\title{
Trombectomía fármaco-mecánica en trombosis venosa profunda aguda con el método de Ascher en el Hospital Médica Campestre de León, Guanajuato
}

\author{
Pharmacomechanical thrombectomy in acute deep vein \\ thrombosis with the Ascher method fast track
}

\author{
Rómulo Armenta Flores, ${ }^{*}$ Diego Armenta Villalobos, ${ }^{\ddagger}$ Luis Gerardo Domínguez Carrillo§ \\ Citar como: Armenta FR, Armenta VD, Domínguez CLG. Trombectomía fármaco-mecánica en trombosis \\ venosa profunda aguda con el método de Ascher en el Hospital Médica Campestre de León, Guanajuato.
} Acta Med Grupo Angeles. 2021; 19 (2): 272-275. https://dx.doi.org/10.35366/100454

Resumen

Se presenta el primer caso en el Hospital Médica Campestre de León, Guanajuato de trombectomía fármaco-mecánica con la técnica de Ascher que permite resolver la trombosis venosa profunda iliofemoral aguda en una sola sesión.

Palabras clave: Trombosis venosa profunda, trombólisis intratrombo, trombectomía fármaco-mecánica, AngioJet.

\section{INTRODUCCIÓN}

La trombosis venosa profunda (TVP) proximal (iliofemoral) aguda es una condición vascular potencialmente grave (siendo una emergencia cuando se presenta phlegmasia alba dolens o bien phlegmasia cerúlea dolens), que requiere evacuación del coágulo a la brevedad para evitar y/o reducir la incidencia de un embolismo pulmonar (EP) y disminuir la intensidad del síndrome postrombótico (SPT), como se ha

* Cirujano Cardiovascular, División de Cirugía del Hospital Médica Campestre. León, Guanajuato, México.

₹ Estudiante de Medicina. Facultad de Medicina de la Universidad de Guanajuato, Campus León. México.

§specialista en Medicina de Rehabilitación. Catedrático de la Facultad de Medicina de la Universidad de Guanajuato, Campus León. México.

Correspondencia:

Dr. Rómulo Armenta Flores

Correo electrónico: roarflo17@gmail.com

Aceptado: 15-06-2020.

www.medigraphic.com/actamedica

\section{Abstract}

The first report of one case of pharmaco-mechanical thrombectomy with the Ascher technique, resolving an acute iliofemoral deep vein thrombosis in a single session was at the Hospital Médica Campestre located in Leon; Mexico.

Keywords: Deep venous thrombosis, intra-thrombus thrombolysis, pharmacomechanical thrombectomy, AngioJet.

referido ampliamente en la literatura médica reciente. ${ }^{1,2}$ La TVP iliofemoral se asocia con severas repercusiones a corto y largo plazo, tanto física, psicológica y económicamente en más del $40 \%$ de los pacientes que son tratados sólo con anticoagulación. ${ }^{3}$ En la actualidad, hay métodos que han mejorado de manera sustancial la evolución de estos casos. La trombólisis intratrombo y la trombectomía fármacomecánica han demostrado una mejoría en la calidad de vida, permeabilidad de la vena tratada por disminución en la carga del trombo dentro de la vena afectada y disminución del edema; teniendo como objetivo la remoción del trombo y disminuir la gravedad del SPT. ${ }^{4-6}$

\section{CASO CLÍNICO}

Mujer de 41 años, gesta 4, con 21 días en puerperio postcesárea; siete días antes de su ingreso a urgencias, inició con aumento de volumen y dolor al deambular en miembro pélvico izquierdo, progresivo de distal a proximal, con calificación 8/10 en EVA (escala visual análoga), impidiendo la ambulación; mejorando con elevación de extremidades pélvicas en decúbito dorsal. La exploración inicial mostró paciente femenino consciente y bien orientada, coopera- 
dora y con facies de dolor, signos vitales. TA 140/85; FC 80X'; FR 20X'; peso $85.00 \mathrm{~kg}$, talla $168 \mathrm{~cm}$; IMC 30.1, aumento de volumen de miembro pélvico izquierdo con diferencia de $+3 \mathrm{~cm}$ en pierna y muslo respecto a la contralateral, sin cambios de coloración, pulsos presentes y llenado capilar normal. El laboratorio mostró: citometría hemática, química sanguínea (12 elementos), pruebas de coagulación y examen general de orina sin alteraciones; el dímero $\mathrm{D}$ con valores de $1,000 \mathrm{ng} / \mathrm{mL}$ (valor de referencia $500 \mathrm{ng} / \mathrm{mL}$ ), telerradiografía de tórax sin datos patológicos, el ultrasonido Doppler venoso (Figura 1) de miembro pélvico izquierdo con trombosis venosa profunda desde vena poplítea hasta vena iliaca común izquierda, por lo que se inició manejo con clexane de 80 mg subcutánea c/12 horas, se ingresó a piso y se programó para realizar trombectomía fármaco-mecánica. Durante su estancia en el piso (12 horas) se infundieron $3 \mathrm{~L}$ de solución fisiológica y media hora antes de iniciar el procedimiento se pasó una carga de $500 \mathrm{~mL}$ de solución fisiológica, se colocó sonda Foley $12 \mathrm{Fr}$ con bolsa recolectora de orina.

\section{DESCRIPCIÓN DE TÉCNICA UTILIZADA}

El procedimiento se efectuó en sala de hemodinamia, con la paciente en decúbito ventral, con asistencia de sedación + anestesia local. La vía de acceso fue a través de la vena safena menor izquierda con control ultrasonográfico.

Se administró un bolo de $3 \mathrm{mil}$ UI de heparina no fraccionada, colocando un introductor $8 \mathrm{Fr}$, pasando una guía hidrofílica de $0.035 \times 260 \mathrm{~cm}$ hasta vena cava inferior, además de un catéter Imager II 5 Fr cobra 2 (Boston Scientific) hasta vena cava inferior infrarrenal y paso de medio de contraste diluido al $50 \%$, para definir extensión del trombo (Figura 2A), el cual llegó hasta la vena iliaca común izquierda. Se realizó previa preparación de consola de Angiojet Ultra con 15 mg de alteplasa en $100 \mathrm{~mL}$ de solución fisiológica.
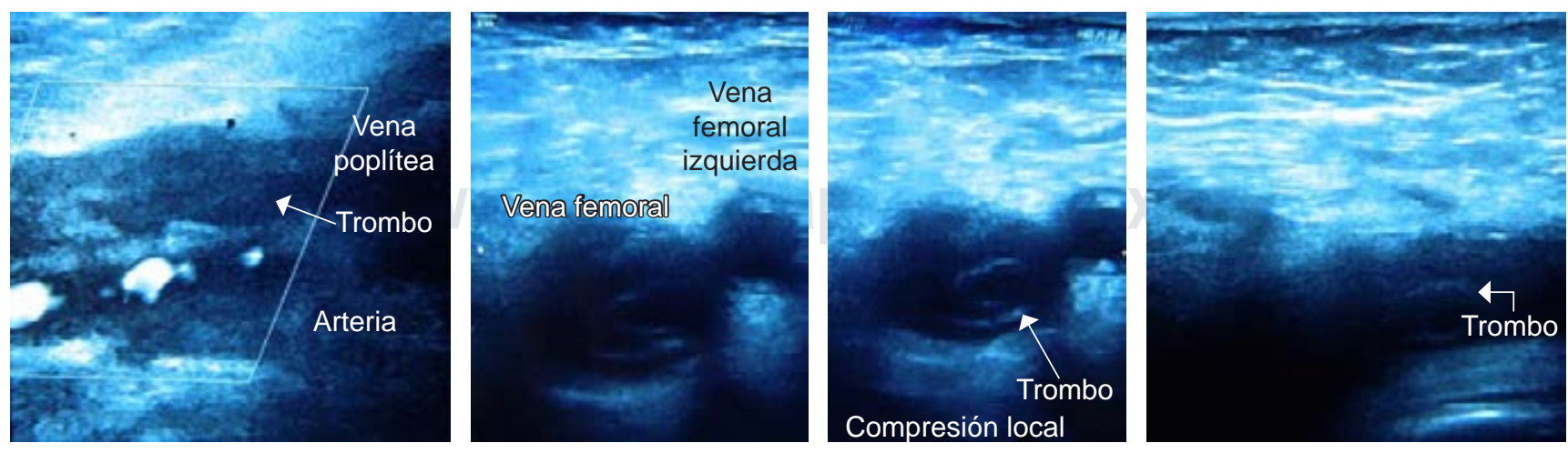

Figura 1: Ultrasonido Doppler dúplex que muestra trombosis venosa profunda (TVP) de vena poplítea a iliaca izquierda.
Se introdujo catéter Zelante DVT 7 Fr (Boston Scientific) con colocación de punta del catéter proximal al trombo, activado en modo irrigación (Figura 2B) (Power-pulse). Después se retiró catéter Zelante de proximal a distal (vena iliaca común a vena poplítea) para trombólisis.

Se maceró el trombo usando un balón no complaciente XXL 14/40 mm (Boston Scientific) inflando por segmentos de proximal a distal a $5 \mathrm{mmHg}$ (Figura 2C). Se retiró el balón, pasaron 20 minutos para esperar la actividad del trombolítico.

Se reintrodujo el catéter Zelante hasta la vena iliaca y se activó el modo de extracción del coágulo trombolizado, utilizando 120 segundos por recorrido (total dos recorridos en 240 segundos); obteniendo abundante coágulo lisado en la bolsa recolectora del AngioJet Ultra.

Se realizó flebografía de control, logrando permeabilidad $100 \%$ y flujo reestablecido al compararse con flebografía inicial (Figura 3).

Se retiraron catéter de flebografía y guía, colocando un Tegaderm de $2.0 \mathrm{~cm}$ en el sitio de acceso además de vendaje compresivo con venda Elastomedic de $15 \mathrm{~cm}$ de ancho, desde base de los dedos a tercio medio de muslo. La paciente pasó a piso con dieta y control de líquidos, los flujos de orina $50 \mathrm{~cm}^{3} /$ hora, sin cambio de coloración. Una hora después del procedimiento, se administró una ámpula de $5 \mathrm{mg}$ de fondaparinux subcutánea en dosis única.

Después de 24 horas de estancia hospitalaria y retiro de sonda Foley, la paciente egresó con indicaciones de reposo relativo, dieta normal, rivaroxabán $15 \mathrm{mg} \mathrm{VO}$ c/12 horas.

En vista de revisión en consulta externa a siete días de postoperatorio, la paciente se encontró asintomática, deambulando, con simetría del diámetro de extremidades pélvicas, sin hematoma en sitio de punción en área poplítea izquierda. La paciente continuó con anticoagulación extendida con rivaroxabán $15 \mathrm{mg} 1 \times 1$, reintegrándola a sus actividades cotidianas. 
Figura 2:

Flebografía con paciente en decúbito prono. A) Trombosis venosa profunda poplítea-iliaca izquierda mostrando una guía

hidrofílica hasta vena cava inferior, además de un catéter Imager II 5 Fr cobra 2, hasta vena cava inferior infrarrenal y paso de medio de contraste diluido al $50 \%$, para definir extensión del trombo. B) Se muestra catéter

Zelante DVT 7 Fr con punta del catéter proximal al trombo, activado en modo irrigación. C) Balón no complaciente inflando por segmentos de proximal a distal a $5 \mathrm{mmHg}$ para maceración del trombo.
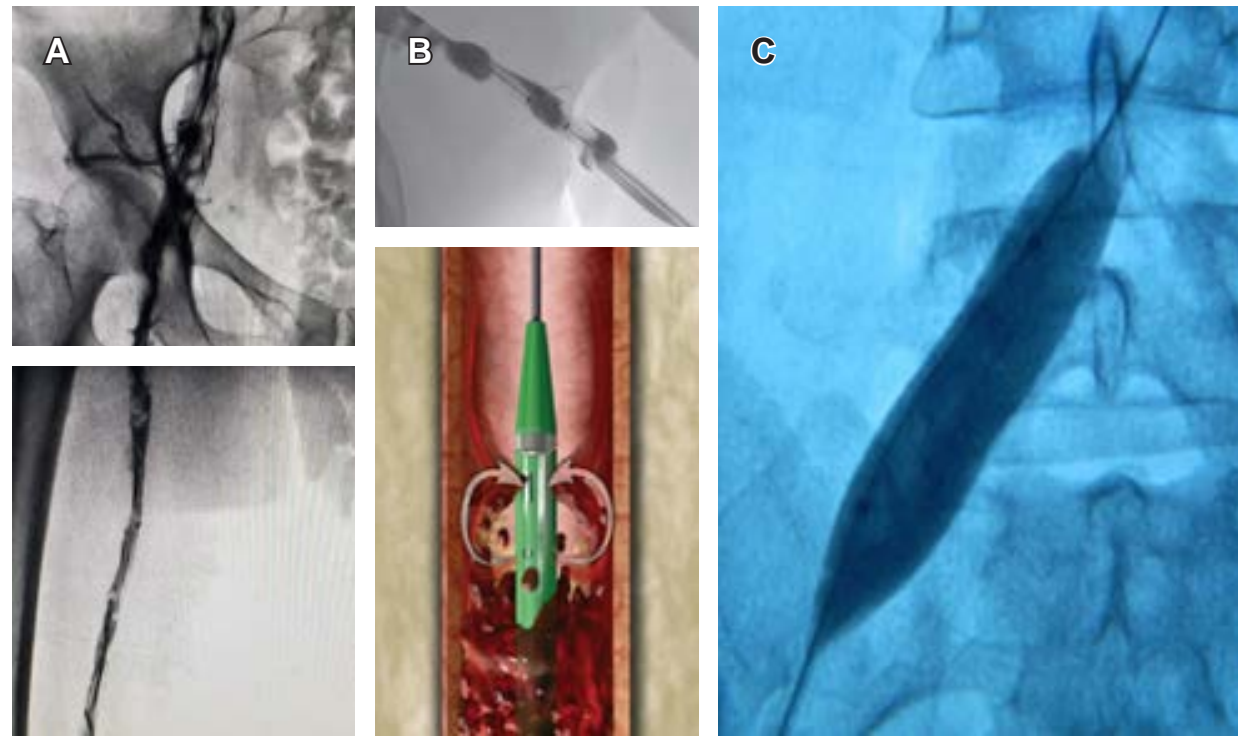

\section{DISCUSIÓN}

La trombólisis fármaco-mecánica es una tecnología que combina la trombólisis intratrombo y la trombectomía reolítica mecánica, que ha demostrado seguridad y eficacia, así como disminución del riesgo de sangrado, al compararla con la trombólisis intratrombo. 6,7 Sin embargo, algunos autores han cuestionado estos beneficios, argumentando que estas técnicas requieren varias sesiones e infusiones repetidas de trombolítico, lo que se asocia con estancias hospitalarias prolongadas que incrementan el costo y el riesgo de complicaciones. ${ }^{5-8}$

Para evitar todo lo anterior, el grupo del Hospital Langone de Brooklyn NY desarrolló un protocolo que disminuye el número de sesiones, la cantidad de trombolítico infundido y la estancia prolongada en estos pacientes. En este trabajo, reportamos el primer paciente tratado por nuestro grupo en León, Guanajuato utilizando dicho protocolo y se revisan los detalles técnicos que permiten reproducir los buenos resultados obtenidos por el grupo de Ascher. ${ }^{9}$ Aunque es una técnica que tiene en función al menos nueve años, y que al menos en los últimos dos años ya se cuenta con un catéter más potente para ser utilizado en venas de mayor calibre, no se había utilizado en la localidad.

Al realizar este caso, seguimos en su mayoría el protocolo descrito por Ascher y colaboradores con algunos cambios prácticos y adecuados a nuestro medio, por ejemplo: 1) anticoagulación sistémica luego del diagnóstico inicial con heparina fraccionada, en lugar de no fraccionada; 2) se utilizó un solo introductor $8 \mathrm{Fr}$ en vez de varios 5 a $8 \mathrm{Fr}$; 3) se usaron $15 \mathrm{mg}$ de alteplasa por el tamaño del trombo (poplítea a iliaca común), en este caso sólo se aplicó una insuflación al balón para maceración del trombo fresco, logrando la desaparición de éste, mostrada en la venografía de control. Al respecto, Ascher recomienda repetir la angioplastia las veces necesarias hasta obtener flujo sin restricción; 4) en nuestro caso, esperamos 20 minutos antes de realizar la aspiración del trombo para dar tiempo al trombolítico (Ascher recomienda de 10 a $30 \mathrm{~min}$, dependiendo del tiempo de evolución de la TVP); 5) en nuestro medio, no él. A su vez, Ascher no lo utilizó por la misma razón. ${ }^{6}$ En el caso reportado, se recuperó la permeabilidad del $100 \%$ y al inflar el balón no mostró disminuciones de diámetro, por lo que se descartó cualquier obstrucción en la región iliofemoral tratada (Ascher al no disponer de UIV en su serie, reporta obstrucciones proximales en $20 \%$ de sus casos al inflar el balón de angioplastia, por lo que utilizó férulas [Wallstent] para restablecer permeabilidad en todos sus pacientes). ${ }^{7}$ En el postoperatorio inmediato nosotros utilizamos una sola dosis de fondaparinux (Arixtra) $5 \mathrm{mg}$ antes de egresar a la paciente con anticoagulación oral (rivaroxabán), a diferencia de Ascher, quien utiliza heparina no fraccionada $\times 12$ horas. Debe anotarse que para el éxito del procedimiento, los casos deben ser elegidos con cuidado; al analizar la serie de Ascher, observamos que en su protocolo la diferencia con otros reportes está en la maceración del trombo con el balón de angioplastia de baja presión, lo que permite aumentar el área de contacto del trombo, facilita el contacto con el trombolítico y fractura el coágulo en partes pequeñas, por lo que se aspira con mayor facilidad y, al insuflar el balón, constata si hay o no obstrucción significativa.

Por último, como Ascher menciona, no utilizamos filtro de vena cava inferior, ya que su uso no es rutinario en TVP se utilizó ultrasonido intravascular (UIV) por no disponer de 

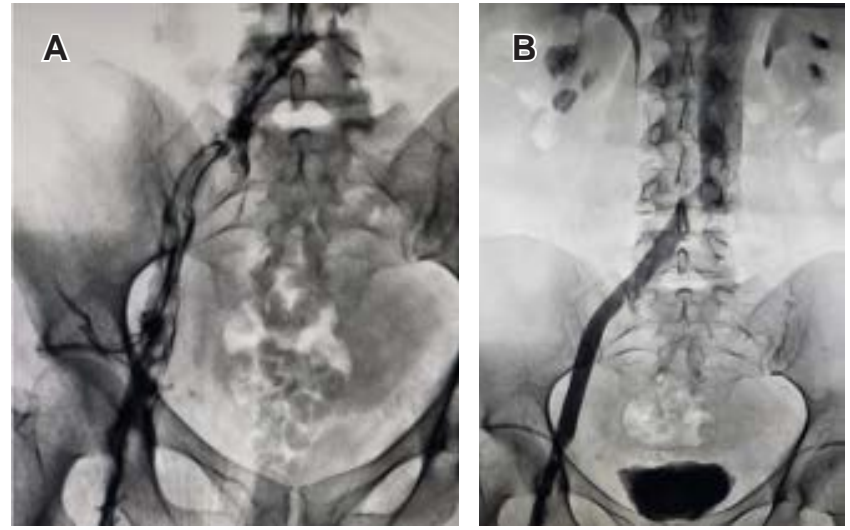

Figura 3: Flebografía con paciente en decúbito prono. A) Trombosis venosa profunda poplítea-iliaca izquierda. B) Flujo venoso femoro-iliaco restablecido.

iliofemoral sin contraindicación para anticoagulación, el cual tiene indicación ante la presencia de un trombo flotante en la vena cava inferior. ${ }^{1,5}$

En los últimos 20 años se utilizó inicialmente la trombólisis sistémica, posteriormente la técnica regional intratrombo, hasta llegar a la utilización de diferentes artefactos para evacuar el coágulo; una de las técnicas más recientes es la trombectomía fármaco-mecánica, la cual con el refinamiento tecnológico, ha demostrado su efectividad al grado de poder remover el coágulo de forma ambulatoria. En el caso de esta investigación, utilizamos la técnica descrita por Ascher y colaboradores y reportada en $2019,{ }^{9}$ y la escogimos por ser una técnica asequible con los recursos disponibles en nuestro país y por los buenos resultados obtenidos en su serie. El término fast-track lo utiliza Ascher cuando sólo hay una sesión y el paciente egresa el mismo día; en nuestro caso, la egresamos al día siguiente por cuestiones administrativas, pero podría haber egresado en el postoperatorio inmediato. Con este método, el uso de trombolítico es con dosis baja y, por tanto, se disminuye el riesgo de sangrado como lo reportó Wong $\mathrm{P}$ y su equipo. ${ }^{6}$ Nuestro caso no presentaba comorbilidad (con sobrepeso en puerperio), por lo que resultó candidata para este método.

\section{CONCLUSIÓN}

Reportamos el primer caso de nuestra serie en León, Guanajuato de TVP iliofemoral aguda resuelto con técnica de Ascher (fast-track) trombólisis fármaco-mecánica, que permite optimizar recursos para beneficio del paciente.

\section{REFERENCIAS}

1. Meissner MH, Gloviczki P, Comerota AJ, Dalsing MC, Eklof BG, Gillespie $\mathrm{DL}$ et al. Early thrombus removal strategies for acute deep venous thrombosis: clinical practice guidelines of the Society for Vascular Surgery and the American Venous Forum. J Vasc Surg. 2012; 55 (5): 1449-1462.

2. Ng TT, Sigman M, Weaver FA. Basic data related to thrombolytic therapy for acute venous thrombosis. Ann Vasc Surg. 2014; 28 (4): 1039-1044.

3. Comerota AJ, Gravett MH. Iliofemoral venous thrombosis. J Vasc Surg. 2007; 46 (5): 1065-1076.

4. Armenta FR, Sánchez LF, Ramírez CE. Trombólisis intratrombo simultánea en tromboembolia pulmonar y trombosis venosa profunda. Acta Med. 2018; 16 (2): 170-172.

5. Vedantham S, Goldhaber SZ, Julian JA, Kahn SR, Jaff MR, Cohen DJ et al. Pharmacomechanical catheter-directed thrombolysis for deepvein thrombosis. N Engl J Med. 2017; 377 (23): 2240-2252.

6. Wong PC, Chan YC, Law Y, Cheng SWK. Percutaneous mechanical thrombectomy in the treatment of acute iliofemoral deep vein thrombosis: a systematic review. Hong Kong Med J. 2019; 25 (1): 48-57.

7. Comerota AJ, Kearon C, Gu CS, Julian JA, Goldhaber SZ, Kahn SR et al. Endovascular thrombus removal for acute iliofemoral deep vein thrombosis. Circulation. 2019; 139 (9): 1162-1173.

8. Nathan AS, Giri J. Reexamining the open-vein hypothesis for acute deep venous thrombosis. Circulation. 2019; 139 (9): 1174-1176.

9. Ascher E, Chait J, Pavalonis A, Marks N, Hingorani A, Kibrik P. Fasttrack thrombolysis protocol: A single-session approach for acute iliofemoral deep venous thrombosis. J Vasc Surg Venous Lymphat Disord. 2019; 7 (6): 773-780. 